\title{
Computing Majority by Constant Depth Majority Circuits with Low Fan-in Gates*†
}

\author{
Alexander S. Kulikov ${ }^{1}$ and Vladimir V. Podolskii ${ }^{2}$ \\ 1 Steklov Mathematical Institute, Russian Academy of Sciences, St. Petersburg, \\ Russia \\ kulikov@pdmi.ras.ru \\ 2 Steklov Mathematical Institute, Russian Academy of Sciences, St. Petersburg, \\ Russia; and \\ National Research University, Higher School of Economics, St. Petersburg, \\ Russia \\ podolskii@mi.ras.ru
}

\begin{abstract}
We study the following computational problem: for which values of $k$, the majority of $n$ bits $\mathrm{MAJ}_{n}$ can be computed with a depth two formula whose each gate computes a majority function of at most $k$ bits? The corresponding computational model is denoted by $\mathrm{MAJ}_{k} \circ \mathrm{MAJ}_{k}$. We observe that the minimum value of $k$ for which there exists a $\mathrm{MAJ}_{k} \circ \mathrm{MAJ}_{k}$ circuit that has high correlation with the majority of $n$ bits is equal to $\Theta\left(n^{1 / 2}\right)$. We then show that for a randomized $\mathrm{MAJ}_{k} \circ \mathrm{MAJ}_{k}$ circuit computing the majority of $n$ input bits with high probability for every input, the minimum value of $k$ is equal to $n^{2 / 3+o(1)}$. We show a worst case lower bound: if a $\mathrm{MAJ}_{k} \circ \mathrm{MAJ}_{k}$ circuit computes the majority of $n$ bits correctly on all inputs, then $k \geq n^{13 / 19+o(1)}$. This lower bound exceeds the optimal value for randomized circuits and thus is unreachable for pure randomized techniques. For depth 3 circuits we show that a circuit with $k=O\left(n^{2 / 3}\right)$ can compute $\mathrm{MAJ}_{n}$ correctly on all inputs.
\end{abstract}

1998 ACM Subject Classification F.1.3 Complexity Measures and Classes

Keywords and phrases circuit complexity, computational complexity, threshold, majority, lower bound, upper bound

Digital Object Identifier 10.4230/LIPIcs.STACS.2017.49

\section{Introduction}

In this paper we study majority functions and circuits consisting of them. These functions and circuits arise for various reasons in many areas of Computational Complexity (see e.g. $[12,14,7])$. In particular, the iterated majority function (or recursive majority) consisting of iterated application of majority of small number of variables to itself, turns out to be of great importance, helps in various constructions and provides an example of the function with interesting complexity properties in various models $[8,11,13,9]$.

One of the most prominent examples to illustrate this is the proof by Valiant [17] that the majority $\mathrm{MAJ}_{n}$ of $n$ variables can be computed by a boolean circuit of depth $5.3 \log n$.

\footnotetext{
* The full version of this paper is available as ECCC report TR16-158 - https://eccc.weizmann.ac.il/ report/2016/158/.

$\dagger$ The research presented in Section 4 was supported by Russian Science Foundation (project 16-11-10123). The research presented in Section 5 was partially supported by grant MK-7312.2016.1 and by the Russian Academic Excellence Project '5-100'.
} 
The construction of Valiant is randomized and there is no deterministic construction known achieving the same (or even reasonably close) depth parameter. The construction works as follows. Consider a uniform boolean formula (that is, tree-like circuit) consisting of $5.3 \log n$ interchanging layers of AND and OR gates of fan-in 2. For each input to the circuit substitute a random variable of the function $\mathrm{MAJ}_{n}$. Valiant showed that this circuit computes $\mathrm{MAJ}_{n}$ with positive probability. Note that AND and OR gates are precisely $\mathrm{MAJ}_{2}$ functions with different threshold values. Thus this construction can be viewed as a computation of $\mathrm{MAJ}_{n}$ by a circuit consisting of $\mathrm{MAJ}_{2}$ gates. There are versions of this construction with the circuits consisting of $\mathrm{MAJ}_{3}$ gates (see, e.g., [5]).

In this paper we study what happens with this setting if we restrict the depth of the circuit to a small constant. That is, we study for which $k$ the function $\mathrm{MAJ}_{n}$ can be computed by small depth circuit consisting of $\mathrm{MAJ}_{k}$ gates. We mostly concentrate on depth 2 and denote the corresponding model by $\mathrm{MAJ}_{k} \circ \mathrm{MAJ}_{k}$. For example, the majority of $n=7$ bits $x_{1}, x_{2}, \ldots, x_{7}$ can be computed with the following $\mathrm{MAJ}_{k} \circ \mathrm{MAJ}_{k}$ circuit for $k=5$ :

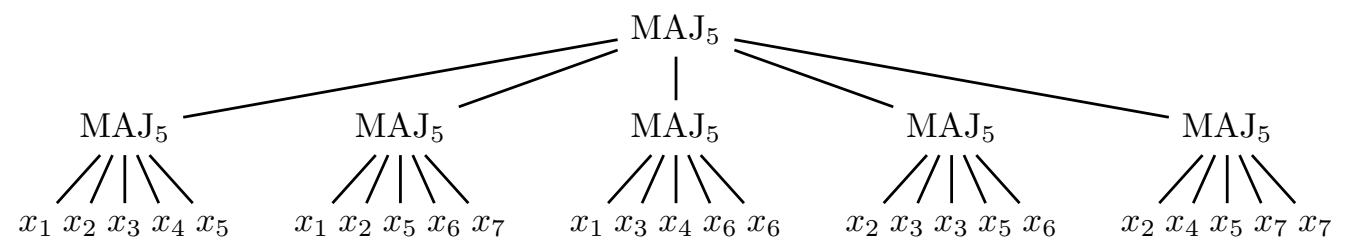

We study which upper and lower bounds on $k$ can be shown.

More context to the problem under consideration comes from the studies of boolean circuits of constant depth. The class $\widehat{T C}^{0}$ of boolean functions computable by polynomial size constant depth circuits consisting of MAJ gates plays one of the central roles in this area. Its natural generalization is the class $T C^{0}$ in which instead of MAJ gates one can use arbitrary linear threshold gates, that is analogs of the majorities in which variables are summed up with arbitrary integer coefficients and are compared with arbitrary integer threshold. It is known that to express any threshold function it is enough to use exponential size coefficients. To show that $\mathrm{TC}^{0}$ is actually the same class as $\widehat{T C}^{0}$ it is enough to show that any linear threshold function can be computed by constant depth circuit consisting of threshold functions with polynomial-size coefficients (polynomial size can be simulated in $\widehat{T C}^{0}$ by repetition of variables). It was shown by Siu and Bruck in [16] that any linear threshold function can be computed by polynomial size depth-3 majority circuit. This result was improved to depth-2 by Goldmann, Håstad and Razborov in [4]. More generally, it was shown in [4] that depth- $d$ polynomial size threshold circuit can be computed by depth- $(d+1)$ polynomial size majority circuit, in particular establishing the class of depth-2 threshold circuits as one of the weakest classes for which we currently do not know superpolynomial size lower bounds. The best lower bound known so far is $\Omega\left(\frac{n^{3 / 2}}{\log ^{3} n}\right)$ by Kane and Williams [10].

Note, however, that the result of [4] does not translate to monotone setting. Hofmeister in [6] showed that there is a monotone linear threshold function requiring exponential size depth-2 monotone majority circuit. Recently this result was extended by Chen, Oliveira and Servedio [2] to monotone majority circuits of arbitrary constant depth.

Our setting can be viewed as a scale down of the setting of [4] and [6]. In [4, 6] exponential weight threshold functions are compared to depth-2 threshold circuits with polynomial weights. In our setting we compare weight- $n$ threshold functions with depth- 2 threshold circuits with weights $k$. In this paper we consider monotone setting.

Another context to our studies comes from the studies of lower bounds against $\widehat{\mathrm{TC}}^{0}$. Allender and Koucký in [1] showed that to prove that some function is not in $\widehat{\mathrm{TC}}^{0}$ it is enough 
to show that some self-reducible function requires circuit-size at least $n^{1+\varepsilon}$ when computed by constant depth majority circuit. As an intermediate result they show that $\mathrm{MAJ}_{n}$ can be computed by $O(1)$-depth circuit consisting of $\mathrm{MAJ}_{n^{\varepsilon}}$ gates and of size $O(n \log n)$. This setting is similar to ours, however in this paper we are interested in the precise depth and we do not pose additional bounds on the size of the circuit (however note that the bound on the fan-in $k$ of the gates and the bound on the depth $d$ of the circuit naturally imply the bound of $O\left(k^{d}\right)$ on the size of the circuit).

We consider three models of computation of the majority function: computation on most of the inputs (that is, high correlation with the function), randomized computation with small error probability on all inputs, and deterministic computation with no errors. We prove the following lower and upper bounds for our setting.

Circuits with high correlation. We observe that the minimum value of $k$ for which there exists a $\mathrm{MAJ}_{k} \circ \mathrm{MAJ}_{k}$ circuit that computes $\mathrm{MAJ}_{n}$ correctly on $2 / 3$ fraction of all the inputs, is equal to $\Theta\left(n^{1 / 2}\right)$. A lower bound is proved by observing that a circuit with $k=\alpha n^{1 / 2}$ does not even have a possibility to read a large fraction of input bits when the constant $\alpha$ is small enough. We show that in this case the circuit errs on many inputs. An upper bound is proved for the following natural circuit: pick $k=\Theta\left(n^{1 / 2}\right)$ random subsets of the $n$ inputs bits of size $k$, compute the majority for each of them, and then compute the majority of results. Such a circuit computes $\mathrm{MAJ}_{n}$ correctly with high probability on inputs whose weight is not too close to $n / 2$. By tuning the parameters appropriately, we ensure that the middle layers of the boolean hypercube (containing inputs where the circuits errs with high probability) constitute only a small fraction of all the inputs.

Randomized circuits. We prove that for a probabilistic distribution $\mathcal{C}$ of $\mathrm{MAJ}_{k} \circ \mathrm{MAJ}_{k}$ circuits with a property that for every input $A \in\{0,1\}^{n}$ the probability that $\mathcal{C}(A)=\operatorname{MAJ}_{n}(A)$ is $1-\varepsilon$ for a constant $\varepsilon>0$, the minimum value of $k$ is $n^{2 / 3}$, up to polylogarithmic factors. A lower bound is proved by showing that a small circuit must err on a large fraction of minterms/maxterms of $\mathrm{MAJ}_{n}$. Roughly, the majority function have many inputs $A \in\{0,1\}^{n}$ with a property that changing a single bit in $A$ changes the value of the function (these are precisely minterms and maxterms of $\mathrm{MAJ}_{n}$ ). If $k$ is small enough, a $\mathrm{MAJ}_{k} \circ \mathrm{MAJ}_{k}$ circuit can reflect such a change in the value only for a small fraction of inputs. To show an upper bound, we split the $n$ input bits into blocks and for each block compute several middle layers values of the bits of this block in sorted order. We then compute the majority of all the resulting values. We show that by tuning the parameters appropriately, one can ensure that this circuit err only on a polynomially small fraction of inputs.

Deterministic circuits. The trivial upper bound on $k$ is $k \leq n$. We do not have any nontrivial upper bound on $k$ for depth 2 circuits. We however have examples for $n=7,9,11$ of circuits with $k=n-2$. For depth 3 we have an upper bound $O\left(n^{2 / 3}\right)$ which coincides with the optimal value for depth 2 randomized circuits up to polylogarithmic factor. We prove this upper bound by extending the construction of upper bound for depth 2 randomized circuits. We use an extra layer of the circuit to preorder the inputs. Regarding the lower bound for depth 2 we observe that the following simple special case cannot compute $\mathrm{MAJ}_{n}$ : each gate is a standard majority (that is, with threshold $k / 2$ ) of exactly $k=n-2$ distinct variables. Next, we proceed to the main result of the paper. We show that the minimum value of $k$ for which there is a depth 2 circuit computing MAJ $_{n}$ on all inputs is at least $n^{13 / 19}$ up to a polylogarithmic factor. 
Note that this lower bound exceeds the optimal value of $k$ for randomized circuits. Thus, despite the fact that randomized techniques is extensively used for studying majority and circuits constructed from it and proves to be very powerful (recall for example Valiant's result [17]), in our setting using combinatorial methods we prove a lower bound that is unreachable for a pure probabilistic approach. The proof of this result however is still probabilistic: in essence we consider a circuit with $k$ smaller than $n^{13 / 19}$ and build a distribution on inputs that fools this circuit. The catch is that the distribution is tailored to fool this particular circuit: it is constructed via a non-trivial process that involves the values of the gates of the circuit on various inputs.

The rest of the paper is organized as follows. In Section 2 we give necessary definitions and collect technical statements. In Section 3 we study circuits computing the function with high correlation. In Section 4 we give bounds for randomized circuits. In Section 5 we study deterministic circuits. Finally, in Section 6 we give concluding remarks and state several open problems.

\section{Definitions and Preliminaries}

In this section we will give necessary definitions and collect technical statements that we will use throughout the paper.

We are going to study circuits computing the well known boolean majority function defined as follows: $\operatorname{MAJ}_{n}\left(x_{1}, x_{2}, \ldots, x_{n}\right)=\left[\sum_{i=1}^{n} x_{i} \geq n / 2\right]$. Here, [.] denotes the standard Iverson bracket: for a predicate $P,[P]=1$ if $P$ is true, and $[P]=0$ is $P$ is false. To abuse notation, we will also use $[m]$ to denote the set $\{1,2, \ldots, m\}$.

It will be convenient to use $X=\left\{x_{1}, x_{2}, \ldots, x_{n}\right\}$ for the set of $n$ input bits. For an assignment $A: X \rightarrow\{0,1\}$, by $w(A)$ we denote the weight of $A$, that is, $\sum_{x \in X} A(x)$. For a subset of input variables $S \subseteq X$, by $w_{S}(A)$ we denote the weight of $A$ on $X: w_{S}(A)=$ $\sum_{x \in S} A(x) \cdot \operatorname{By}_{\operatorname{MAJ}_{S}}(X)$ we denote the majority function on $S: \operatorname{MAJ}_{S}(X)=\left[\sum_{x \in S} x \geq\right.$ $|S| / 2]$. In particular, $\mathrm{MAJ}_{X}$ is just $\mathrm{MAJ}_{n}$.

An assignment $A: X \rightarrow\{0,1\}$ is called a minterm of $\operatorname{MAJ}_{n}$ if $\operatorname{MAJ}_{n}(A)=1$, but flipping any 1 to 0 in $A$ results in an assignment $A^{\prime}$ such that $\operatorname{MAJ}_{n}\left(A^{\prime}\right)=0$. A maxterm is defined similarly with the roles of 0 and 1 interchanged.

The majority function is a special case of a threshold function: $f(X)=\left[\sum_{i=1}^{n} a_{i} x_{i} \geq t\right]$. For such a function $f$ and an assignment $A: X \rightarrow\{0,1\}$, let difference of $f$ w.r.t. $A$ be $\operatorname{diff}(f, A)=\sum_{i=1}^{n} a_{i} A\left(x_{i}\right)-t$. In particular, $f(A)=1 \operatorname{iff} \operatorname{diff}(f, A) \geq 0$.

$\mathrm{The} \mathrm{MAJ}_{k} \circ \mathrm{MAJ}_{k}$ computational model that we study in this paper is defined as a depth two formula (we will call it a circuit also) consisting of arbitrary threshold gates of the form $\left[\sum c_{i} x_{i} \geq t\right]$ where $c_{i}$ 's are positive integers (this, in particular, means that the model is monotone) and $\sum c_{i} \leq k$. At the same time, abusing notation, by $\mathrm{MAJ}_{n}$ and $\mathrm{MAJ}_{X}$ we always mean the standard majority function. We note that the coefficients in $c_{i}$ can be simulated by repetition of variables (note that $k$ upper bounds the sum of the coefficients). So the generalization of the $\mathrm{MAJ}_{k}$ in the circuit compared to $\mathrm{MAJ}_{n}$ is that we allow arbitrary threshold. We note however, that if we are interested in the value of $k$ up to a constant factor (which we usually do), it is not an actual generalization since any threshold can be simulated by substituting constants 0 and 1 as inputs to the circuit.

For a gate $G$ at the bottom level of a $\mathrm{MAJ}_{k} \circ \mathrm{MAJ}_{k}$ circuit, by $X(G)$ we denote the set of its input bits. 


\subsection{Tail Bounds and Binomial Coefficients Estimates}

We will use the following versions of Chernoff-Hoeffding bound (see, e.g., [3]).

- Lemma 1 (Chernoff-Hoeffding bound). Let $Y=\sum_{i=1}^{m} Y_{i}$, where $Y_{i}, i \in[m]$, are independently distributed in $[0,1]$. Then for all $t>0, \operatorname{Pr}[Y>E[Y]+t], \operatorname{Pr}[Y<E[Y]-t] \leq e^{-2 t^{2} / m}$. For all $\varepsilon>0, \operatorname{Pr}[Y>(1+\varepsilon) E[Y]], \operatorname{Pr}[Y<(1-\varepsilon) E[Y]] \leq e^{-\frac{\varepsilon^{2}}{3} E[Y]}$.

We will also need the following well known estimates for the binomial coefficients (see, e.g., [15, Section 4.2]):

- Lemma 2. The middle binomial coefficient is about $n^{1 / 2}$ times smaller than $2^{n}$. To make it smaller than $2^{n}$ by arbitrary polynomial factor, it is enough to step away from the middle by about $\Theta(\sqrt{n \ln n})(0<c<1$ is a constant below):

$$
\left(\begin{array}{c}
n \\
n / 2
\end{array}\right)=\Theta(1) \cdot 2^{n} \cdot n^{-1 / 2} \quad \text { and } \quad\left(\begin{array}{c}
n \\
\frac{n}{2}+\frac{c \sqrt{n \ln n}}{2}
\end{array}\right)=\Theta\left(2^{n} n^{-\frac{1}{2}} n^{-\frac{c^{2}}{2}}\right) .
$$

\subsection{Hypergeometric Distribution}

The hypergeometric distribution is defined in the following way. Consider a set $S$ of size $m$ and its subset $S^{\prime}$ of size $k$. Select (uniformly) a random subset $T$ of size $t$ in $S$. Then a random variable $\left|T \cap S^{\prime}\right|$ has a hypergeometric distribution. The values $m, k$ and $t$ are parameters here. We will need the following basic properties of this distribution.

- Lemma 3. Suppose in hypergeometric distribution $k=k(m) \leq m / 2$ (that is, $k$ may depend on $m)$. Let $t=t(m)$ be a function with $\varepsilon m<t<(1-\varepsilon) m$ for some constant $0<\varepsilon<1$. Then, for any integer $l, \operatorname{Prob}\left(\left|T \cap S^{\prime}\right|=l\right)=O\left(k^{-1 / 2}\right)$, where $O(\cdot)$ is for $m \rightarrow \infty$ and the constant inside $O(\cdot)$ depends on $\varepsilon$, but does not depend on $m, k$ and $t$. Moreover, if $\left|l-\frac{t k}{m}\right|=O(1)$, then this probability is in fact $\Theta\left(k^{-1 / 2}\right)$.

- Lemma 4. Suppose in hypergeometric distribution $k=k(m) \leq m / 2$ (that is, $k$ may depend on $m)$. Let $t=t(m)$ be a function with $\varepsilon m<t<(1-\varepsilon) m$ for some constant $0<\varepsilon<1$. Consider an arbitrary antichain $A$ on $S^{\prime}$ (that is, a family of subsets of $S^{\prime}$ none of which is a subset of some other $)$. Then the probability $\operatorname{Pr}\left[T \cap S^{\prime} \subseteq A\right]=O\left(k^{-1 / 2}\right)$, where $O(\cdot)$ is for $m \rightarrow \infty$ and the constant inside $O(\cdot)$ depends on $\varepsilon$, but does not depend on $m, k$ and $t$.

- Lemma 5. For $S, S^{\prime}$ and $T$ as above we have $\operatorname{Prob}\left\{\left|T \cap S^{\prime}\right| \geq l\right\} \leq(t k / m)^{l}$.

\section{Circuits with High Correlation}

In this section, we prove that the minimum value of $k$ for which there exists a $\mathrm{MAJ}_{k} \circ \mathrm{MAJ}_{k}$ circuit that computes $\mathrm{MAJ}_{n}$ correctly on, say, 2/3 fraction of all the inputs, is equal to $\Theta\left(n^{1 / 2}\right)$.

\subsection{Upper Bound}

- Theorem 6. For any $\varepsilon>0$, there exists a circuit $C$ in $\mathrm{MAJ}_{k} \circ \mathrm{MAJ}_{k}$, where $k=O_{\varepsilon}\left(n^{1 / 2}\right)$, that agrees with $\mathrm{MAJ}_{n}$ on at least $(1-\varepsilon)$ fraction of the boolean hypercube $\{0,1\}^{n}$.

Proof Sketch. The required circuit is straightforward: we just pick $k$ random subsets $S_{1}, S_{2}, \ldots, S_{k}$ of $X$ of size $k$, compute the majority for each of them, and then compute the majority of the results: $C(X)=\operatorname{MAJ}_{k}\left(\operatorname{MAJ}_{S_{1}}(X), \operatorname{MAJ}_{S_{2}}(X), \ldots, \operatorname{MAJ}_{S_{k}}(X)\right)$. The 
resulting circuit has a high probability of error on middle layers of the boolean hypercube. We however select the parameters so that all the inputs from these middle layers constitute only a small $\varepsilon / 2$ fraction. We then show that among all the remaining inputs (not belonging to middle layers) there is only a fraction $\varepsilon / 2$ (of all the inputs) where $\mathrm{MAJ}_{n}$ may be computed incorrectly. Overall, this gives a circuit that errs on at most $\varepsilon$ fraction of the inputs.

\subsection{Lower Bound}

- Theorem 7. Let $C$ be a $\mathrm{MAJ}_{k} \circ \mathrm{MAJ}_{k}$ circuit that computes $\mathrm{MAJ}_{n}$ correctly on a fraction $1-\varepsilon$ of all $2^{n}$ inputs for a constant $\epsilon \leq 1 / 3$. Then $k=\Omega_{\varepsilon}\left(n^{1 / 2}\right)$.

Proof Sketch. Let $k=\alpha n^{1 / 2}$ for a small enough constant $\alpha=\alpha(\varepsilon)$. Note that such a circuit can read at most $k^{2}=\alpha^{2} n$ of the input bits. This means that the circuit errs on a large number of inputs.

\section{$4 \quad$ Randomized Circuits}

The upper bound from the previous section, however, is not enough to obtain a randomized circuit since the construction in Theorem 6 has a very high error probability on the middle layers of the boolean cube. By a randomized circuit here we mean a probabilistic distribution on deterministic circuits computing the function correctly on every input with high probability.

It is not difficult to see that the existence of a randomized circuit is equivalent to an existence of a deterministic circuit computing the function correctly on most of minterms and maxterms.

- Lemma 8. If there exists a randomized circuit $\mathcal{C}$ in $\mathrm{MAJ}_{k} \circ \mathrm{MAJ}_{k}$ computing $\mathrm{MAJ}_{n}$ with error probability $\varepsilon$, then there exists a deterministic circuit $C$ in $\mathrm{MAJ}_{k} \circ \mathrm{MAJ}_{k}$ computing $\mathrm{MAJ}_{n}$ incorrectly on at most $\varepsilon$ fraction of minterms and maxterms. Conversely, if there exists a deterministic circuit $C$ in $\mathrm{MAJ}_{k} \circ \mathrm{MAJ}_{k}$ computing $\mathrm{MAJ}_{n}$ incorrectly on at most $\varepsilon$ fraction of minterms and maxterms, then there exists a randomized circuit $\mathcal{C}$ in $\mathrm{MAJ}_{k} \circ \mathrm{MAJ}_{k}$ computing $\mathrm{MAJ}_{n}$ with error probability at most $2 \varepsilon$.

From now on instead of probabilistic circuits we study deterministic circuits with high accuracy on two middle layers of $\{0,1\}^{n}$.

\subsection{Upper Bound}

- Theorem 9. There exists a randomized $\mathrm{MAJ}_{k} \circ \mathrm{MAJ}_{k}$ circuit computing $\mathrm{MAJ}_{n}$ incorrectly on each input with probability at most $1 / \operatorname{poly}(n)$ for $k=O\left(n^{2 / 3} \log ^{1 / 2} n\right)$.

Proof Sketch. Partition the set of $n$ input bits into $n^{1 / 3}$ blocks of size $p=n^{2 / 3}: X=$ $X_{1} \sqcup X_{2} \sqcup \ldots \sqcup X_{\frac{n}{p}}$. For each block $X_{i}$, compute $\left[\sum_{x \in X_{i}} x \geq m\right]$ for all $m \in\left[\frac{p}{2}-\frac{t}{2}, \frac{p}{2}+\frac{t}{2}\right]$ for $t \approx n^{1 / 3} \log ^{1 / 2} n$, and return the majority of results. By selecting the right value of $t$, this gives a circuit that computes $\mathrm{MAJ}_{n}$ incorrectly only on a fraction $\frac{1}{\operatorname{poly}(n)}$ of inputs.

\subsection{Lower Bound}

In this subsection we show that the upper bound of the previous subsection is essentially tight.

- Theorem 10. If a $\mathrm{MAJ}_{k} \circ \mathrm{MAJ}_{k}$ circuit computes $\mathrm{MAJ}_{n}$ on a $1-\varepsilon$ fraction of minterms and maxterms for $\varepsilon<1 / 10$, then $k=\Omega\left(n^{2 / 3}\right)$. 
Proof Sketch. The majority function have many inputs $A \in\{0,1\}^{n}$ with a property that changing a single bit in $A$ changes the value of the function (these are precisely minterms and maxterms of $\mathrm{MAJ}_{n}$ ). If $k=\alpha n^{2 / 3}$ for a small enough constant $\alpha$, a $\mathrm{MAJ}_{k} \circ \mathrm{MAJ}_{k}$ circuit can reflect such a change in the value only for a small fraction of inputs.

\section{Deterministic Circuits}

In this section, we consider $\mathrm{MAJ}_{k} \circ \mathrm{MAJ}_{k}$ circuits that compute $\mathrm{MAJ}_{n}$ correctly on all $2^{n}$ inputs.

\subsection{Upper Bounds}

\subsubsection{Depth Two}

In this section, we present $\mathrm{MAJ}_{k} \circ \mathrm{MAJ}_{k}$ circuits computing MAJ $\mathrm{J}_{n}$ on all inputs for $k=n-2$ when $n=7,9,11$. These circuits were found by extensive computer experiments (with the help of SAT-solvers). Though the examples below look quite "structured", currently, we do not know how to generalize them to all values of $n$ (not to say about constructing such circuits for sublinear values of $k$ ). In the examples below, we provide $k=n-2$ sequences consisting of $k=n-2$ integers from $[n]$. These are exactly the input bits of the $k$ majority gates at the lower level of the circuit. That is, each gate computes the standard $\mathrm{MAJ}_{k}$ function (whose threshold value is $k / 2$ ).

\begin{tabular}{|c|c|}
\hline$n=7:$ & $n=9$ \\
\hline 12345 & 1234567 \\
\hline $\begin{array}{lllll}12 & 3 & 6 & 7\end{array}$ & 1234589 \\
\hline 14567 & 1236789 \\
\hline 22456 & $\begin{array}{lllllll}1 & 4 & 5 & 6 & 7 & 8 & 9\end{array}$ \\
\hline $\begin{array}{lllll}3 & 4 & 5 & 7\end{array}$ & $\begin{array}{lllllll}1 & 3 & 5 & 5 & 7 & 9 & 9\end{array}$ \\
\hline & 12446688 \\
\hline
\end{tabular}

$n=11:$

$\begin{array}{lllllllll}1 & 2 & 3 & 4 & 5 & 6 & 7 & 8 & 9\end{array}$

$\begin{array}{llllllllll}1 & 2 & 3 & 4 & 5 & 6 & 7 & 10 & 11\end{array}$

$\begin{array}{llllllllll}1 & 2 & 3 & 4 & 5 & 8 & 9 & 10 & 11\end{array}$

$\begin{array}{llllllllll}1 & 2 & 3 & 6 & 7 & 8 & 9 & 10 & 11\end{array}$

$\begin{array}{llllllllll}1 & 4 & 5 & 6 & 7 & 8 & 9 & 10 & 11\end{array}$

$122 \quad 2 \quad 4 \quad 6 \quad 6 \quad 8 \quad 1010$

$2 \begin{array}{lllllllll}2 & 4 & 4 & 5 & 6 & 7 & 8 & 10 & 11\end{array}$

$\begin{array}{llllllllll}3 & 3 & 5 & 5 & 7 & 7 & 8 & 9 & 11\end{array}$

$\begin{array}{lllllllll}3 & 3 & 6 & 8 & 9 & 9 & 9 & 10 & 10\end{array}$

Note that in the examples above there is always a gate in the circuit having one variable repeated more than once. Next we observe that this is unavoidable for $k=n-2$.

- Lemma 11. For odd $n$ there is no $\mathrm{MAJ}_{k} \circ \mathrm{MAJ}_{k}$ circuit for $k=n-2$ with all gates being standard majorities (that is, with the threshold $n / 2$ ) and having exactly $k$ distinct variables in each gate on the bottom level.

\subsubsection{Depth Three}

In this section we extend the proof of the upper bound for randomized depth-2 circuits (Theorem 9) to construct a circuit of depth 3 for $k=O\left(n^{2 / 3}\right)$ computing majority on all inputs.

- Theorem 12. For $k=O\left(n^{2 / 3}\right)$ there is a circuit of depth 3 computing majority of $n$ variables on all inputs.

Proof Sketch. We adopt the strategy of the proof of Theorem 9. That is, we break inputs into $O\left(n^{1 / 3}\right)$ blocks, compute majorities on each block on middle $O\left(n^{1 / 3}\right)$ layers and then 
compute the majority of the results. We use the third layer of majority gates to induce additional structure on the inputs.

\subsection{Lower Bound}

In this section we will extend the lower bound on $k$ above $\Omega\left(n^{2 / 3}\right)$ for depth- 2 circuits computing $\mathrm{MAJ}_{n}$ on all inputs.

- Theorem 13. Suppose a $\mathrm{MAJ}_{k} \circ \mathrm{MAJ}_{k}$ circuit computes $\mathrm{MAJ}_{n}$ on all inputs. Then $k=\Omega\left(n^{13 / 19} \cdot(\log n)^{-2 / 19}\right)$.

We also show the following result for the special case of circuits with bounded weights.

- Theorem 14. Suppose a $\mathrm{MAJ}_{k} \circ \mathrm{MAJ}_{k}$ circuit computes $\mathrm{MAJ}_{n}$ on all inputs and uses only weights at most $W$ in the gates. Then $k=\Omega\left(n^{7 / 10} \cdot(\log n)^{-1 / 5} \cdot W^{-3 / 10}\right)$.

In particular, we get the following corollary for circuits with unweighted gates.

- Corollary 15. Suppose a $\mathrm{MAJ}_{k} \circ \mathrm{MAJ}_{k}$ circuit computes $\mathrm{MAJ}_{n}$ on all inputs and each variable occurs in each gate of the bottom level at most once. Then $k=\Omega\left(n^{7 / 10} \cdot(\log n)^{-1 / 5}\right)$.

The rest of this section is devoted to the unified proof of these lower bounds. To follow this proof it is convenient to think that $k=n^{\frac{2}{3}+\varepsilon}$ for some small $\varepsilon>0$. In the end it will indeed be the case up to a logarithmic factor. In the proof we will calculate everything precisely in terms of parameters $n$ and $k$, but we will provide estimates assuming that $k=n^{2 / 3+\varepsilon}$. This is done in order to help the reader to follow the proof.

Let $F$ be a $\mathrm{MAJ}_{k} \circ \mathrm{MAJ}_{k}$ formula computing $\mathrm{MAJ}_{n}$ on all inputs from $\{0,1\}^{n}$. Denote by $W$ the largest weight of a variable in gates of $F$.

\subsubsection{Normalizing a formula}

We start by "normalizing" $F$, that is, removing some pathological gates from $F$. We do this in two consecutive stages.

Stage 1: removing AND-like gates. We will need that no gate can be fixed to 0 by assigning a small number of variables to 0 (here and in what follows we consider gates from the bottom level only). For this, assume that there is a gate that can be fixed to 0 by assigning to 0 less than $n /(100 k)=n^{1 / 3-\varepsilon} / 100$ variables. Take these variables and substitute them by 0 ; this kills this gate (and might potentially introduce new gates with the property). We repeat this process until there are no bad gates left. Recall that the number of gates at the bottom level is at most $k=n^{2 / 3+\varepsilon}$, so there are at most $k=n^{2 / 3+\varepsilon}$ steps in this process and hence $n$ is replaced by $99 n / 100$. To simplify the presentation, we just assume that $|X|=n$ and that $F$ has no bad gates.

Stage 2: removing other pathological gates and variables. The formula $F$ contains at most $k^{2}=n^{\frac{4}{3}+2 \varepsilon}$ occurrences of variables (counting with multiplicities). Let $x^{*} \in X$ be a least frequent variable at the leaves. The number of occurrences of $x^{*}$ is at most $k^{2} / n=n^{1 / 3+2 \varepsilon}$. In the following we consider only assignments $A$ with $\operatorname{diff}\left(\mathrm{MAJ}_{n}, A\right)=-1 \operatorname{setting} x^{*}$ to 0 :

$$
\mathcal{A}^{*}=\left\{A: X \rightarrow\{0,1\} \mid \operatorname{diff}\left(\mathrm{MAJ}_{n}, A\right)=-1 \text { and } A\left(x^{*}\right)=0\right\} .
$$

We also focus on the gates from the first level that depend on $x^{*}$, denote this set by $\mathcal{G}^{*}$ (hence $\left|\mathcal{G}^{*}\right| \leq k^{2} / n=n^{1 / 3+2 \varepsilon}$ ). The total number of variables in the gates from $\mathcal{G}^{*}$ (counting with multiplicities) is at most $k\left|\mathcal{G}^{*}\right| \leq k^{3} / n=n^{1+3 \varepsilon}$. 
We now additionally normalize the circuit. We get rid of the following bad gates and variables:

1. gates in $\mathcal{G}^{*}$ that can be assigned to 1 by fixing less than $n^{2} /\left(100 k^{2}\right)=n^{2 / 3-2 \varepsilon} / 100$ variables in $X \backslash\left\{x^{*}\right\}$ to 1 ;

2. gates in $\mathcal{G}^{*}$ with the weight of the variable $x^{*}$ greater than $100 k^{3} / n^{2}=100 n^{3 \varepsilon}$;

3. variables with total weight in all gates in $\mathcal{G}^{*}$ greater than $100 k^{3} / n^{2}=100 n^{3 \varepsilon}$.

We do this by the following iterative procedure. If on some step we have a gate violating 1 we fix less than $n^{2} /\left(100 k^{2}\right)=n^{2 / 3-2 \varepsilon} / 100$ variables of the gate among $X \backslash\left\{x^{*}\right\}$ to 1 to assign the gate to a constant. If we have a gate violating 2 we fix all the variables of the gate among $X \backslash\left\{x^{*}\right\}$ to 1 to assign the gate to a constant. If we have a variable violating 3 , we fix the violating variable to 1 .

We note that if we fix all variables in $G \in \mathcal{G}^{*}$ except $x^{*}$ to 1 , then the gate becomes constant. Indeed, if it is not constant, then the gate outputs 0 on the input with $x^{*}=0$ and the rest of the variables equal to 1 . Due to the monotonicity of the gate this means that the gate can be assigned to 0 by assigning a single variable $x^{*}$ to 0 and we got rid of the gates with this property on the first stage of the normalization.

Since there are at most $k^{2} / n=n^{1 / 3+2 \varepsilon}$ gates in $\mathcal{G}^{*}$ we will fix at most $n / 100$ variables for case 1 . Since the total weight of $x^{*}$ is at most $k^{2} / n=n^{1 / 3+2 \varepsilon}$ we will have case 2 at most $n /(100 k)=n^{1 / 3-\varepsilon} / 100$ times. Since each gate has at most $k=n^{2 / 3+\varepsilon}$ variables we will fix at most $n / 100$ variables for the second case. Since the total weight of all variables in $\mathcal{G}^{*}$ is at most $k^{3} / n=n^{1+3 \varepsilon}$ we will fix at most $n / 100$ of them for the case 3 .

In particular, we have fixed all variables having weight greater than $100 k^{3} / n^{2}=100 n^{3 \varepsilon}$ in some gate of $\mathcal{G}^{*}$, so from now on we can assume that $W \leq 100 k^{3} / n^{2}$.

Another important observation is that now in each gate there are at least $n^{2} /\left(100 k^{2}\right)$ inputs. Otherwise the gate falls under condition of case 1 above.

After this normalization $n$ is replaced by $97 n / 100$. To simplify the presentation, again, we assume that $|X|=n$ and the circuit $F$ is normalized. Note that after redefining $n$ the threshold of the function $\mathrm{MAJ}_{n}$ we are computing is no longer $n / 2$, but rather is $c n$ for some constant $c$ close to $1 / 2$. This does not affect the computations in the further proof.

\subsubsection{Analysis}

The key idea is that if we have an assignment $A \in \mathcal{A}^{*}$ with $\operatorname{diff}\left(\mathrm{MAJ}_{n}, A\right)=-1$, then there is a gate $G \in \mathcal{G}^{*}$ with $-W \leq \operatorname{diff}(G, A) \leq-1$. Indeed, otherwise we can flip the variable $x^{*}$, the value of $\mathrm{MAJ}_{n}$ changes, but none of the gates changes their value. The plan of the proof is to construct an assignment that violates this condition. This will lead to a contradiction.

For an assignment $A \in \mathcal{A}^{*}$ with $\operatorname{diff}\left(\mathrm{MAJ}_{n}, A\right)=-1$ and integer parameters $s$ and $d$ (to be chosen later), consider the following process walk $(A, s, d)$.

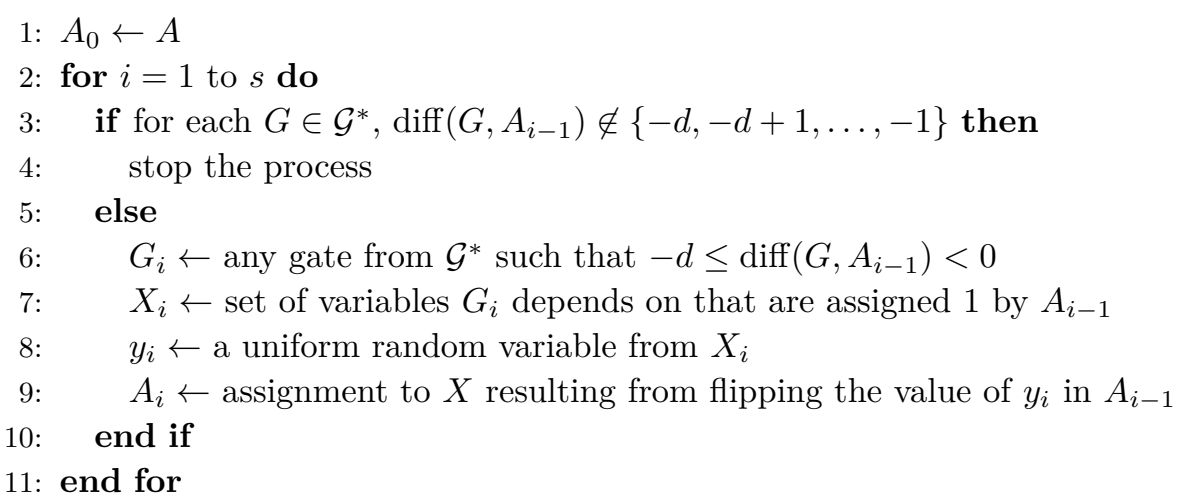


Clearly, this process decreases the weight of the initial assignment $A$ by 1 at each iteration, for at most $s$ iterations. In particular, $w(A)-w\left(A_{i}\right)=i$. We now consider three cases.

Case 1. There exists an assignment $A \in \mathcal{A}^{*}$ with $\operatorname{diff}\left(\mathrm{MAJ}_{n}, A\right)=-1$ such that walk $(A, s, d)$ stops after less than $s$ iterations for some choices of random bits. This means that after $t<s$ iterations, for all the gates $G$ in $\mathcal{G}^{*}$ we have that either $\operatorname{diff}\left(G, A_{t}\right)<-d$, or $\operatorname{diff}\left(G, A_{t}\right) \geq 0$.

We select randomly a subset $T$ of $t$ variables from $Z=\left\{x \in X \backslash\left\{x^{*}\right\}: A_{t}(x)=0\right\}$ and flip them. Denote the resulting assignment by $A^{\prime}$. Clearly, $w(A)=w\left(A^{\prime}\right)$ and so $\operatorname{diff}\left(\mathrm{MAJ}_{n}, A^{\prime}\right)=-1$. Therefore there must be a gate $G$ in $\mathcal{G}^{*}$ such that $-W \leq \operatorname{diff}\left(G, A^{\prime}\right)<$ 0 . Thus, before flipping $t$ random variables, all the gates with negative difference has difference less than $-d$, while after the flipping, at least one gate $G$ has difference at least $-W$. Let $Z^{\prime}=\left\{x \in X(G) \backslash\left\{x^{*}\right\}: A_{t}(x)=0\right\}$. This means that the flipping changed the values of at least $r=(d-W) / W$ variables of $G$, that is, $\left|T \cap Z^{\prime}\right| \geq r$.

Let $p$ be the probability that $\left|T \cap Z^{\prime}\right| \geq r$ where the probability is taken over the random choice of $T$. By choosing the parameters $s$ and $d$ we will make $p$ small enough so that with non-zero probability no gate from $\mathcal{G}^{*}$ satisfies this. Due to the discussion above this leads to a contradiction since flipping $x^{*}$ changes the value of the function, but not the value of the circuit. The probability that no gate from $\mathcal{G}^{*}$ satisfies $\left|T \cap Z^{\prime}\right| \geq r$ is at least $1-\left|\mathcal{G}^{*}\right| p$. The probability $p$ can be upper bounded using Lemma 5: $p \leq\left(\frac{t\left|Z^{\prime}\right|}{|Z|}\right)^{r} \leq\left(\frac{s k}{n / 2}\right)^{r}$ where the second inequality follows since $t<s,\left|Z^{\prime}\right| \leq k$ and $|Z| \geq \frac{n}{2}$.

We want the probability $1-\left|\mathcal{G}^{*}\right| p$ to be positive. Since $\left|\mathcal{G}^{*}\right| \leq k^{2} / n=n^{1 / 3+2 \varepsilon}$ we get the following inequality on $s, d$, and $k:\left(k^{2} / n\right) \cdot(2 s k / n)^{r}<1$. We can satisfy this if $s k<n / 4$ and $r \geq \log \frac{k^{2}}{n}$. Since $\log n>\log \frac{k^{2}}{n}$ for the latter it is enough to have $d=W \log n$. Overall, this case poses the following constraint for the considered parameters:

$$
s k \leq n / 4 .
$$

Case 2. For each assignment $A \in \mathcal{A}^{*}$ (i.e., $\left.\operatorname{diff}\left(\mathrm{MAJ}_{n}, A\right)=-1\right)$ the process walk $(A, s, d)$ goes through all $s$ iterations for all choices of random bits. We consider two subcases here.

Case 2.1. For each assignment $A \in \mathcal{A}^{*}$ (i.e., $\operatorname{diff}\left(\mathrm{MAJ}_{n}, A\right)=-1$ ) there exists a choice of variables $y_{1}, \ldots, y_{s}$ at line 8 of the process walk $(A, s, d)$, such that for each gate $G \in$ $\left\{G_{1}, \ldots, G_{s}\right\}$ (recall that the gates $G_{1}, \ldots, G_{s}$ are selected at line 6 of the process) we have $\operatorname{diff}(G, A) \leq f$, where $f$ is again a positive parameter to be chosen later.

We estimate the expected number $E$ of gates $G$ from $\mathcal{G}^{*}$ that have $-d \leq \operatorname{diff}(G, A) \leq f$ where the expectation is taken over the random choices of $A$. Note that a particular gate $G \in \mathcal{G}^{*}$ may appear in the sequence $G_{1}, \ldots, G_{s}$ at most $d$ times: the first time it appears, it must have $\operatorname{diff}\left(G, A_{1}\right) \leq-1$ for the current assignment $A_{1}$, the next time it has $\operatorname{diff}\left(G, A_{2}\right) \leq-2$ for the new current assignment $A_{2}$, and so on. If $E d<s$ we get a contradiction: take an assignment $A \in \mathcal{A}^{*}$ with $\operatorname{diff}\left(\mathrm{MAJ}_{n}, A\right)=-1$ such that the number of gates $G$ in $\mathcal{G}^{*}$ with $-d \leq \operatorname{diff}(G, A) \leq f$ is at most $E$, then we cannot have that for all of $G_{1}, \ldots, G_{s}$ it is true that $-d \leq \operatorname{diff}\left(G_{i}, A\right) \leq f$, there are just not enough gates with this diff.

Now we upper bound $E$. Due to the normalization stage any fixed gate has at least $n^{2} /\left(100 k^{2}\right)=n^{2 / 3-2 \varepsilon} / 100$ variables in it. Note that the set of inputs $B$ to the gate $G$ that give $\operatorname{diff}(G, B)=i$ for any $i$ form an antichain. Then due to Lemma 4 the probability for a gate to attain a certain value is at most $O(k / n)=O\left(1 / n^{1 / 3-\varepsilon}\right)$. 
Hence

$$
E \leq\left|\mathcal{G}^{*}\right| \cdot(f+d) \cdot O\left(\frac{k}{n}\right)=\frac{k^{2}}{n} \cdot(f+d) \cdot O\left(\frac{k}{n}\right)=O\left(\frac{k^{3}(f+d)}{n^{2}}\right)=O\left(\frac{k^{3} f}{n^{2}}\right)
$$

where for the last equality we add the constraint

$$
d=O(f)
$$

Overall, this case poses the following constraint for the parameters:

$$
O\left(\frac{k^{3} f d}{n^{2}}\right)=O\left(f d n^{3 \varepsilon}\right)<s .
$$

Case 2.2. There exists an assignment $A \in \mathcal{A}^{*}$ (i.e., $\operatorname{diff}\left(\mathrm{MAJ}_{n}, A\right)=-1$ ) such that for any choice of variables $y_{1}, \ldots, y_{s}$, for at least one gate $G \in\left\{G_{1}, \ldots, G_{s}\right\}$ we have $\operatorname{diff}(G, A)>f$.

Fix a gate $G \in \mathcal{G}^{*}$ with $\operatorname{diff}(G, A)>f$. We are going to upper bound the probability (over the random choices of variables $y_{1}, \ldots, y_{s}$ ) that $G$ appears among $G_{1}, \ldots, G_{s}$ during the process. If this probability is less than $1 / k$, then by the union bound with a positive probability no gate such gate appears among $G_{1}, \ldots, G_{s}$ which leads to a contradiction with the case statement.

For $G$ to appear among $G_{1}, \ldots, G_{s}$, the process has to select a variable appearing in $G$ at line 8 many times. Indeed, if $G$ appears in the process, then its diff with the current assignment is negative. At the same time, in the beginning of the process $\operatorname{diff}(G, A)>f$. Each time when the process reduces a variable at line 8 (that is, changes its value from 1 to 0 ), the value of the linear function computed at $G$ decreases by at most $W$ (just because $W$ is the maximum weight of a variable in all the gates in $\left.\mathcal{G}^{*}\right)$. Thus, it is enough to upper bound the probability that for a fixed gate $G \in \mathcal{G}^{*}$ with $\operatorname{diff}(G, A)>f$, the process selects a variable from $X(G)$ at least $f / W$ times.

Let $Y_{1}, \ldots, Y_{s}$ be random $0 / 1$-variables defined as follows: $Y_{i}=1$ iff the $i$-th reduced variable appears in $G$ (i.e., $\left.y_{i} \in X(G)\right)$. Let $Y=\sum_{i=1}^{s} Y_{i}$. Our goal is to upper bound $\operatorname{Prob}(Y \geq f / W)$

Let $H_{1}, \ldots, H_{l}$ be all the gates that share at least one variable with $G$. Assume that on step $j$ we reduce a variable from $H_{i}$. Then

$$
\operatorname{Prob}\left(Y_{j}=1\right)=\operatorname{Prob}\left(y_{i} \in X(G)\right)=\frac{\left|X(G) \cap X\left(H_{i}\right)\right|}{\left|\left\{x \in X\left(H_{i}\right): A_{j-1}(x)=1\right\}\right|} .
$$

Due to the stage 2.1 of the normalization process, $\left|\left\{x \in X\left(H_{i}\right): A_{j-1}(x)=1\right\}\right| \geq \frac{n^{2}}{100 k^{2}}-d$. To see this, assume the contrary. Recall that $-d \leq \operatorname{diff}\left(H_{i}, A_{j-1}\right)<0$. This means that by increasing at most $d$ variables (i.e., changing their values from 0 to 1 ) from $X\left(H_{i}\right)$ in $A_{j-1}$ results in an assignment of weight at most $\frac{n^{2}}{100 k^{2}}$ that sets $H_{i}$ to 1 . This, in turn, contradicts to the fact that the circuit is normalized. Thus,

$$
\operatorname{Prob}\left(Y_{j}=1\right) \leq \frac{\left|X(G) \cap X\left(H_{i}\right)\right|}{\frac{n^{2}}{100 k^{2}}-d} \leq \frac{\left|X(G) \cap X\left(H_{i}\right)\right|}{\frac{n^{2}}{200 k^{2}}},
$$

where we add a constraint

$$
d \leq \frac{n^{2}}{200 k^{2}} .
$$


We are now going to use the fact that variables from a fixed gate $H_{i}$ can be reduced at most $d$ times. We upper bound $Y=\sum_{i=1}^{s} Y_{i}$ by the following random variable: $Z=$ $\sum_{i=1}^{l} \sum_{j=1}^{d} Z_{i j}$. where each $Z_{i j}$ is a random $0 / 1$-variable such that

$$
\operatorname{Prob}\left(Z_{i j}=1\right)=\frac{\left|X(G) \cap X\left(H_{i}\right)\right|}{\frac{n^{2}}{200 k^{2}}}
$$

and $Z_{i j}$ are independent. That is, instead of reducing variables in some of $H_{i}$ 's in some random order, we reduce $d$ variables in each $H_{i}$. Thus we reduce maximal possible number of variables in all gates. Clearly, for any $r$ we have $\operatorname{Prob}(Y \geq r) \leq \operatorname{Prob}(Z \geq r)$.

Let us bound the expectation of $Z$. Since due to the normalization each variable of $G$ appear in other gates at most $100 k^{3} / n^{2}=100 n^{3 \varepsilon}$ times, we have

$$
\sum_{i, j}\left|X(G) \cap X\left(H_{i}\right)\right| \leq d \cdot\left(100 k^{3} / n^{2}\right) \cdot|X(G)| \leq 100 \cdot d \cdot k^{4} / n^{2}=100 \cdot n^{2 / 3+4 \varepsilon} \cdot W \cdot \log n .
$$

Overall we get $E Z \leq \frac{100 d k^{4} / n^{2}}{n^{2} / 200 k^{2}}=4 \cdot 10^{4} \cdot d \frac{k^{6}}{n^{4}}=4 \cdot 10^{4} \cdot n^{6 \varepsilon} \cdot W \cdot \log n$. Application of Chernoff-Hoeffding bound (Lemma 1) immediately implies that the probability that $Z$ is twice greater than the expectation is exponentially small in $d \cdot \frac{k^{6}}{n^{4}}$. Since $d \cdot \frac{k^{6}}{n^{4}}=W \cdot \log n \cdot n^{9 \varepsilon}$ grows asymptotically faster than $\log n$ for sure, we conclude that $\operatorname{Prob}(Z \geq 2 \cdot E Z)<\frac{1}{n} \leq \frac{1}{k}$. Hence, if $f / W \geq 2 \cdot E Z$, then $\operatorname{Prob}(Y \geq f / W) \leq \operatorname{Prob}(Z \geq 2 \cdot E Z)<\frac{1}{k}$ as desired. Overall, this gives us the following constraint:

$$
f \geq 4 \cdot 10^{4} \cdot d \cdot W \cdot \frac{k^{6}}{n^{4}}=4 \cdot 10^{4} \cdot n^{9 \varepsilon} \cdot W^{2} \cdot \log n .
$$

\subsubsection{Tuning the parameters}

It remains to set the parameters so that the inequalities (2)-(6) are satisfied and $k$ is as large as possible. The inequality (4) sets a lower bound on $s$ in terms of $f$, while (6) sets a lower bound on $f$. Putting them together gives a lower bound on $s: s \geq 4 \cdot 10^{4} \cdot \frac{k^{9}}{n^{6}} \cdot W^{3} \cdot \log ^{2} n$. Combining it with the upper bound on $s$ from (2), we can set the following equality on $k$ and $n: \frac{n}{4 k}=4 \cdot 10^{4} \cdot \frac{k^{9}}{n^{6}} \cdot W^{3} \cdot \log ^{2} n$. Thus $k=\Omega\left(\frac{n^{7 / 10}}{(\log n)^{1 / 5} W^{3 / 10}}\right)$ and it is easy to see that with this $k$ we can pick other parameters to satisfy all the constraints (we set $f$ so that (6) turns into an equality, the inequalities (3) and (5) are satisfied since $\left.W \leq \frac{k^{3}}{n^{2}}\right)$.

This gives a proof of Theorem 14. For $W=1$ we get $k=n^{7 / 10} \cdot(\log n)^{-1 / 5}$, which gives a proof for Corollary 15. For unbounded $W$ recall that we can assume $W \leq \frac{k^{3}}{n^{2}}$ and thus $k=n^{13 / 19} \cdot(\log n)^{-2 / 19}$ and Theorem 13 follows.

\section{Conclusion and Open Problems}

The most interesting question left open is whether one can prove non-trivial upper bounds for $k$ in the worst case. Currently, we do not know how to construct $\mathrm{MAJ}_{k} \circ \mathrm{MAJ}_{k}$ circuits computing $\mathrm{MAJ}_{n}$ on all inputs even for $k=n-2$ (though we have many examples of such circuits for $n=7,9,11$, not to say about $k=n^{\varepsilon}$ for $\varepsilon<1$.

Another natural open question is to get rid of the logarithmic gap between upper and lower bound for depth-2 randomized circuits.

A natural direction is to extend our studies to the case of non-monotone $\mathrm{MAJ}_{k} \circ \mathrm{MAJ}_{k}$ circuits.

Many of our results naturally translate to larger depth circuits. Indeed, note that in the proofs of lower bounds we do not use the fact that the function on the top of the circuit is 
majority. In these proofs it can be any monotone function. Thus we can split a depth- $d$ circuit consisting of $\mathrm{MAJ}_{k}$ into two parts: bottom layer and the rest of the circuit. Then our lower bounds translate to this setting straightforwardly. It is interesting to proceed with the studies of larger depth majority circuits.

Acknowledgments. We would like to thank the participants of Low-Depth Complexity Workshop (St. Petersburg, Russia, May 21-25, 2016) for many helpful discussions.

\section{References}

1 Eric Allender and Michal Koucký. Amplifying lower bounds by means of self-reducibility. J. ACM, 57(3), 2010. doi:10.1145/1706591.1706594.

2 Xi Chen, Igor Carboni Oliveira, and Rocco A. Servedio. Addition is exponentially harder than counting for shallow monotone circuits. Electronic Colloquium on Computational Complexity (ECCC), 22:123, 2015. URL: http://eccc.hpi-web.de/report/2015/123.

3 Devdatt P. Dubhashi and Alessandro Panconesi. Concentration of Measure for the Analysis of Randomized Algorithms. Cambridge University Press, 2009. URL: http://www. cambridge.org/gb/knowledge/isbn/item2327542/.

4 Mikael Goldmann, Johan Håstad, and Alexander A. Razborov. Majority gates VS. general weighted threshold gates. Computational Complexity, 2:277-300, 1992. doi:10.1007/ BF01200426

5 Oded Goldreich. Valiant's polynomial-size monotone formula for majority, 2001. Available at http://www.wisdom.weizmann.ac.il/ oded/PDF/mono-maj.pdf.

6 Thomas Hofmeister. The power of negative thinking in constructing threshold circuits for addition. In Proceedings of the Seventh Annual Structure in Complexity Theory Conference, Boston, Massachusetts, USA, June 22-25, 1992, pages 20-26, 1992. doi:10.1109/SCT. 1992.215377.

7 Stasys Jukna. Boolean Function Complexity - Advances and Frontiers, volume 27 of Algorithms and combinatorics. Springer, 2012. doi:10.1007/978-3-642-24508-4.

8 Stasys Jukna, Alexander A. Razborov, Petr Savický, and Ingo Wegener. On P versus NP cap co-NP for decision trees and read-once branching programs. Computational Complexity, 8(4):357-370, 1999. doi:10.1007/s000370050005.

9 Jesse Kamp and David Zuckerman. Deterministic extractors for bit-fixing sources and exposure-resilient cryptography. SIAM J. Comput., 36(5):1231-1247, 2007. doi:10.1137/ S0097539705446846.

10 Daniel M. Kane and Ryan Williams. Super-linear gate and super-quadratic wire lower bounds for depth-two and depth-three threshold circuits. In Daniel Wichs and Yishay Mansour, editors, Proceedings of the 48th Annual ACM SIGACT Symposium on Theory of Computing, STOC 2016, Cambridge, MA, USA, June 18-21, 2016, pages 633-643. ACM, 2016. URL: http://dl.acm.org/citation. cfm?id=2897518, doi:10.1145/2897518.2897636.

11 Frédéric Magniez, Ashwin Nayak, Miklos Santha, Jonah Sherman, Gábor Tardos, and David Xiao. Improved bounds for the randomized decision tree complexity of recursive majority. Random Struct. Algorithms, 48(3):612-638, 2016. doi:10.1002/rsa. 20598.

12 Marvin Minsky and Seymour Papert. Perceptrons - an introduction to computational geometry. MIT Press, 1987.

13 Elchanan Mossel and Ryan O'Donnell. On the noise sensitivity of monotone functions. Random Struct. Algorithms, 23(3):333-350, 2003. doi:10.1002/rsa.10097.

14 Ryan O'Donnell. Analysis of Boolean Functions. Cambridge University Press, 2014. URL: http://www.cambridge.org/de/academic/subjects/computer-science/ 
algorithmics-complexity-computer-algebra-and-computational-g/ analysis-boolean-functions.

15 Robert Sedgewick and Philippe Flajolet. An introduction to the analysis of algorithms. Addison-Wesley-Longman, 1996.

16 Kai-Yeung Siu and Jehoshua Bruck. On the power of threshold circuits with small weights. SIAM J. Discrete Math., 4(3):423-435, 1991. doi:10.1137/0404038.

17 Leslie G. Valiant. Short monotone formulae for the majority function. J. Algorithms, 5(3):363-366, 1984. doi :10.1016/0196-6774 (84)90016-6. 